\title{
Preoperative C-Reactive Protein as a Risk Factor for Postoperative Delirium in Elderly Patients Undergoing Laparoscopic Surgery for Colon Carcinoma
}

\author{
Dong Xiang, ${ }^{1}$ Hailin Xing, ${ }^{1}$ Huiyu Tai, ${ }^{2}$ and Guozhu Xie ${ }^{1}$ \\ ${ }^{1}$ Department of Anesthesiology, Taizhou People's Hospital, Medical School of Nantong University, Taizhou, China \\ ${ }^{2}$ Department of Intensive Care Unit, Taizhou People's Hospital, Medical School of Nantong University, Taizhou, China \\ Correspondence should be addressed to Guozhu Xie; chentaotg12@163.com
}

Received 4 July 2017; Accepted 24 September 2017; Published 18 October 2017

Academic Editor: Yukio Hayashi

Copyright (C) 2017 Dong Xiang et al. This is an open access article distributed under the Creative Commons Attribution License, which permits unrestricted use, distribution, and reproduction in any medium, provided the original work is properly cited.

Background. Postoperative delirium (POD) is a very common complication in operative disciplines, especially in those elderly patients after cardiac surgery. This study aimed to investigate the relationship between C-reactive protein (CRP) and POD in elderly patients undergoing laparoscopic surgery for colon carcinoma. Methods. 160 elderly patients scheduled to undergo selective laparoscopic surgery for colon carcinoma were prospectively recruited in this present study. The preoperative demographic and medical characteristics, intraoperative variables, and postoperative complications were all recorded in detail. POD assessment was performed once a day for the first 3 days and at 7th day after surgery, respectively. CRP concentrations preoperatively and on postoperative days 1, 2, and 3 were measured by using human enzyme linked immunosorbent assay (ELISA). Results. Of all the 160 enrolled patients, 39 had suffered POD with a POD incidence of $24.4 \%$ within the first week after the operation. The univariate analysis and multiple logistic regression analysis suggested preoperative CRP concentrations as the only independent predicator for POD in patients undergoing laparoscopic surgery for colon carcinoma (OR: 5.87; 95\% CI: 2.22-11.4; $P=0.018$ ). Conclusions. This present study highlighted the predictive role of preoperative CRP concentrations for POD in elderly patients undergoing laparoscopic surgery for colon carcinoma.

\section{Introduction}

Postoperative delirium (POD) is a very common complication in operative disciplines, especially in those elderly patients after cardiac surgery [1]. The described prevalence of POD varies between 30 and $80 \%$ in elderly patients after cardiac surgeries $[2,3]$ and $15 \%-53 \%$ in elderly surgical patients [4]. Numerous studies have revealed that POD is significantly associated with increased complication incidence, long-term cognitive impairment, prolonged hospital length of stay, elevated costs, and overall mortality [5-7]. To predict the implications of POD and improve the quality of care, attempting to determine independent risk factors for POD is of great importance. A number of previous studies have been performed regarding predicative factors for POD; however no consensus has been made until now probably due to the complicated pathogenesis of POD [8]. Previous studies have reported that delirium is associated with elevated proinflammatory cytokines [9] and proteins involved in the stress response [10] in medical or surgical patients. Creactive protein (CRP), one of the most common markers for systemic inflammation, has been indicated as independent risk factor for delirium following vascular surgery [11] and hip surgery [12]. However, the relationship between CRP and POD in patients undergoing laparoscopic surgery for colon carcinoma still remains relatively unknown, which was just the objective of this present study.

\section{Material and Methods}

2.1. Patients. This present study protocol was approved by the Medical Institutional Ethics Committee of Jiangsu province and Taizhou People's Hospital. Those elderly patients (aged $\geq$ 65 years) scheduled to undergo selective laparoscopic surgery 


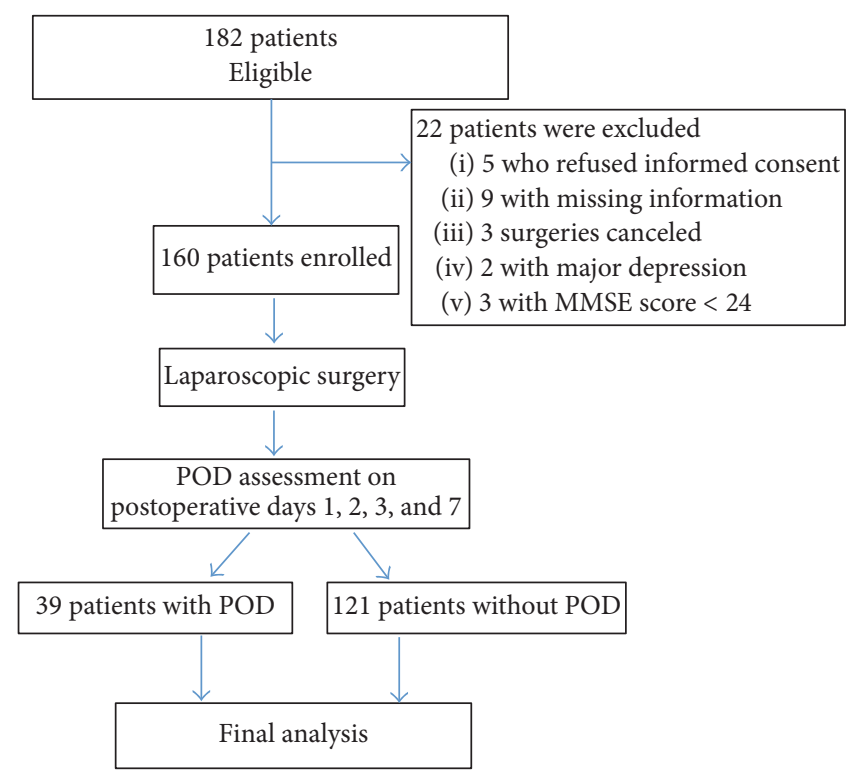

FIgURE 1: Patient CONSORT. MMSE, Mini-Mental State Examination; POD, postoperative delirium.

for colon carcinoma in Taizhou People's Hospital from April, 2014, to January, 2017, were prospectively recruited in this present study. All the participants were required to offer the signed informed consent. Exclusion criteria were described as follows: (1) with major depression; (2) with preexisting or a history of dementia delirium; (3) with cognitive impairment which was defined with a Modified Mini-Mental State Examination (MMSE) score < 24; (4) with clinically neurologic disorder or psychosis. 182 eligible patients were included into our study; 22 of them were excluded for varied reasons (informed consent refusal, missing information, etc.). In total, 160 elderly patients undergoing laparoscopic surgery for colon carcinoma were included into the final analysis, which was shown in the patient CONSORT (Figure 1).

2.2. Methods. Demographic and medical characteristics (including age, gender, and education) were evaluated. The modified Charlson's Comorbidity Index (MCCI) was utilized for the medical comorbidities assessment by summing points [13]. POD was evaluated using the Confusion Assessment Method-Intensive Care Unit (CAM-ICU) by calculating CAM scores [14]. POD assessment was performed once a day (in the evening) for the first 3 days and at 7 th day after surgery, respectively. A positive POD diagnosis was given when patients had a positive result at least for once within 1 week of the assessment. The intraoperative variables (operation time, anesthesia time, blood loss, etc.), postoperative complications (wound infection, urinary tract infection, pulmonary infection, etc.), and postoperative adverse cardiovascular events (such as myocardial infarction, arrhythmias, and heart failure) were also detailed, recorded, and analyzed.

To avoid the interferential impacts by anesthesia, all the enrolled patients underwent the operation under general anesthesia by the same anesthesia team. With no premedication, intravenous midazolam, propofol, sufentanil, and rocuronium were used for inducing anesthesia. Anesthesia was maintained with sevoflurane, propofol, remifentanil, and dexmedetomidine. The serial blood collection preoperatively and on postoperative days 1,2 , and 3 was conducted from all enrolled participants. Blood samples were stored on ice in heparinized tubes and immediately centrifuged $(1500 \mathrm{~g}$ at $4^{\circ} \mathrm{C}$ for 15 minutes). The separated plasma samples from cellular material were then stored at $-80^{\circ} \mathrm{C}$ until assayed. CRP concentrations were measured by using human enzyme linked immunosorbent assay (ELISA) kit (R\&D Systems, Minneapolis, MN, USA). The ELISA was carried out in accordance with the manufacturers' instructions by the same laboratory assistant who was completely blinded to this study.

2.3. Statistical Analysis. The data analysis was performed using SPSS 19.0 (SPSS Inc., Chicago, IL, USA). Categorical data were expressed as number (with percentage, $n \%$ ) and compared with Chi-square test or Fisher exact test. Continuous data were presented as mean levels (with standard deviation) or median (with interquartile range) and compared via the Mann-Whitney $U$-test or Student's $t$-test appropriately. The univariate and multiple logistic regression analyses were plotted to evaluate the predicative validity of pre-, intra-, or postoperative variables for POD. All statistical tests were bilateral probability and $P<0.05$ was considered significant.

\section{Results}

3.1. Preoperative Variables. The demographic and clinical characteristics of the enrolled elderly patients were exhibited in Table 1 in detail. 39 of the 160 patients had suffered POD with a POD incidence of $24.4 \%$, which was similar to other previous studies [15]. The mean age of all the enrolled patients was 70.1 years, and significant difference in age between the patients with or without POD was found. The 
TABLE 1: Pre-, intra-, and postoperative characteristics and plasma CRP levels in patients with or without POD.

\begin{tabular}{|c|c|c|c|}
\hline \multirow{2}{*}{ Variables } & \multicolumn{2}{|c|}{ POD } & \multirow{2}{*}{$P$ value } \\
\hline & Yes $(n=39)$ & No $(n=121)$ & \\
\hline \multicolumn{4}{|l|}{ Preoperative parameters } \\
\hline Age (year) & $72.2 \pm 5.8$ & $69.4 \pm 7.1$ & 0.027 \\
\hline \multicolumn{4}{|l|}{ Gender, $n(\%)$} \\
\hline Male & $23(59.0 \%)$ & $73(60.3 \%)$ & \\
\hline Female & $16(41.0 \%)$ & $48(39.7 \%)$ & 0.881 \\
\hline \multicolumn{4}{|l|}{ Education, $n(\%)$} \\
\hline$\leq$ high school & $27(69.2 \%)$ & $71(58.7 \%)$ & \\
\hline$>$ high school & $12(30.8 \%)$ & $50(41.3 \%)$ & 0.239 \\
\hline BMI $\left(\mathrm{kg} / \mathrm{m}^{2}\right)$ & $21.4 \pm 3.3$ & $22.0 \pm 2.9$ & 0.279 \\
\hline MCCI & $1.6 \pm 0.6$ & $1.4 \pm 0.5$ & 0.041 \\
\hline MMSE score & $25.1 \pm 1.4$ & $25.7 \pm 1.6$ & 0.038 \\
\hline Active smoker, $n(\%)$ & $9(23.1 \%)$ & $30(24.8 \%)$ & 0.828 \\
\hline Heavy drinker, $n(\%)$ & $10(25.6 \%)$ & $14(11.6 \%)$ & 0.032 \\
\hline \multicolumn{4}{|c|}{ ASA physical status, $n(\%)$} \\
\hline I-II & $28(71.8 \%)$ & $80(66.1 \%)$ & \\
\hline III-IV & $11(28.2 \%)$ & $41(33.9 \%)$ & 0.510 \\
\hline \multicolumn{4}{|c|}{ Intraoperative characteristics } \\
\hline Operation time (min) & $203.2 \pm 42.1$ & $182.6 \pm 50.1$ & 0.022 \\
\hline Anesthesia time (min) & $239.7 \pm 55.7$ & $220.3 \pm 51.1$ & 0.045 \\
\hline Blood loss (ml) & $230(50-420)$ & $220(40-490)$ & 0.785 \\
\hline \multicolumn{4}{|l|}{ Lymph node dissection } \\
\hline D2 & $16(41.0 \%)$ & $37(30.6 \%)$ & \\
\hline D3 & $23(59.0 \%)$ & $84(69.4 \%)$ & 0.228 \\
\hline \multicolumn{4}{|l|}{ Operative approach } \\
\hline Multiport surgery & $28(71.8 \%)$ & $63(52.1 \%)$ & \\
\hline Single-port surgery & $11(28.2 \%)$ & $58(47.9 \%)$ & 0.031 \\
\hline \multicolumn{4}{|l|}{ TNM classification } \\
\hline I-II & $24(61.5 \%)$ & $69(57.0 \%)$ & \\
\hline III-IV & $15(38.5 \%)$ & $52(43.0 \%)$ & 0.619 \\
\hline \multicolumn{4}{|l|}{ Postoperative complications } \\
\hline Incision infection & $11(28.2 \%)$ & $16(13.2 \%)$ & 0.030 \\
\hline Urinary tract infection & $4(10.3 \%)$ & $15(12.4 \%)$ & 0.719 \\
\hline Pulmonary infection & $9(23.1 \%)$ & $12(9.9 \%)$ & 0.034 \\
\hline Cardiovascular events & $7(17.9 \%)$ & $7(5.9 \%)$ & 0.019 \\
\hline Bowel obstruction & $6(15.4 \%)$ & $21(17.4 \%)$ & 0.775 \\
\hline Anastomotic leakage & $2(5.13 \%)$ & $7(5.8 \%)$ & 0.877 \\
\hline Postoperative bleeding & $3(7.69 \%)$ & $6(5.0 \%)$ & 0.519 \\
\hline \multicolumn{4}{|l|}{ Plasma CRP levels (mg/L) } \\
\hline Preoperatively & $3.8(0.1-38.5)$ & $2.4(0.1-44.2)$ & 0.011 \\
\hline Postoperative day 1 & $48.7 \pm 14.8$ & $44.9 \pm 16.2$ & 0.196 \\
\hline Postoperative day 2 & $67.5 \pm 20.1$ & $59.7 \pm 19.4$ & 0.032 \\
\hline Postoperative day 3 & $75.1 \pm 18.8$ & $71.5 \pm 21.4$ & 0.349 \\
\hline
\end{tabular}

POD, postoperative delirium; BMI, body mass index; MMSE, Mini-Mental State Examination; MCCI, Modified Charlson Comorbidity Index; ASA, American Society of Anesthesiologists; CRP, C-reactive protein; $P$ values were calculated by Chi-square test, Fisher exact test, Mann-Whitney $U$-test, or Student's $t$-test appropriately. $P<0.05$.

preoperative MCCI score was significantly higher in patients who suffered POD when compared with those without POD. In addition, the preoperative MMSE score and percentage of heavy drinkers were significantly higher in the delirious group than the nondelirious group. No statistically significant differences were found in the gender, education, body mass index, smoking habits, and ASA physical status between the patients with POD or not. 
TABLE 2: Univariate and multivariate logistic regression analyses of POD in patients with colon carcinoma undergoing laparoscopic surgery.

\begin{tabular}{|c|c|c|c|c|}
\hline \multirow{2}{*}{ Variables } & \multicolumn{2}{|c|}{ Univariate } & \multicolumn{2}{|c|}{ Multivariate } \\
\hline & OR $(95 \%$ CI $)$ & $P$ value & OR $(95 \% \mathrm{CI})$ & $P$ value \\
\hline Age & $2.87(1.34-6.63)$ & 0.029 & $1.16(0.96-1.09)$ & 0.46 \\
\hline MCCI & $2.32(1.51-3.23)$ & 0.037 & $1.26(0.49-3.12)$ & 0.61 \\
\hline MMSE score & $2.27(1.14-4.67)$ & 0.011 & $2.01(0.91-4.67)$ & 0.098 \\
\hline Heavy drinker & $1.48(0.89-2.53)$ & 0.15 & & \\
\hline Operative approach & $2.34(0.81-7.22)$ & 0.18 & & \\
\hline Operation time & $2.12(0.62-6.69)$ & 0.29 & & \\
\hline Anesthesia time & $0.97(0.92-1.04)$ & 0.09 & & \\
\hline Wound infection & $2.83(0.62-6.14)$ & 0.25 & & \\
\hline Pulmonary infection & $1.37(0.76-5.19)$ & 0.54 & & \\
\hline Cardiovascular events & $1.28(1.08-1.42)$ & $<0.01$ & $1.11(0.87-1.68)$ & 0.21 \\
\hline \multicolumn{5}{|l|}{ Plasma CRP levels } \\
\hline Preoperative & $11.13(3.51-25.29)$ & $<0.01$ & $5.87(2.22-11.4)$ & 0.018 \\
\hline Postoperative day 2 & $4.54(1.49-13.71)$ & 0.021 & $3.12(0.75-8.27)$ & 0.34 \\
\hline
\end{tabular}

POD, postoperative delirium; MMSE, Mini-Mental State Examination; MCCI, Modified Charlson Comorbidity Index; CRP, C-reactive protein; OR, odds ratio; $\mathrm{CI}$, confidence interval. Multivariate analysis by logistic regression, $P<0.05$.

3.2. Intraoperative Variables. When compared with patients without POD, those with POD showed significantly longer operation time and anesthesia time. As shown in Table 1, the operative approach was also associated with the incidence of POD.

3.3. Postoperative Complications. A significant difference exists between the patients in delirious group or nondelirious group with respect to postoperative incision infection. The incidence of pulmonary infection or cardiovascular events after the surgery seemed significantly associated with the occurrence of POD. When we compared other postoperative complications, no significant differences were observed between groups with respect to the prevalence of urinary tract infection, bowel obstruction, anastomotic leakage, and postoperative bleeding.

3.4. Plasma CRP Concentrations and POD. The postoperative CRP concentrations were many times as high as preoperative levels, which indicated a strong effect of operation on CRP concentrations. The results also revealed that patients with POD showed a significantly higher CRP concentration preoperatively and on postoperative day 2 than those without POD.

3.5. Logistic Regression Analysis for POD. As shown in Table 2, all potential predicative factors mentioned above were summarized by univariate analysis. The results from the univariate logistic regression analysis indicated that age, MCCI, preoperative MMSE score, postoperative cardiovascular events, and CRP concentrations preoperatively or on postoperative day 2 were associated with POD. With these six variables introduced into the final multivariate analysis, the results suggested preoperative CRP concentrations as the independent predicator for POD in patients undergoing laparoscopic surgery for colon carcinoma (OR: 5.87; 95\% CI: $2.22-11.4 ; P=0.018)$.

\section{Discussion}

To our knowledge, this current study demonstrated that plasma CRP concentrations emerged as an independent factor for POD in the elderly patients undergoing laparoscopic surgery for colon carcinoma for the first time. Recently published studies have indicated a positive correlation between POD and early mortality after surgery, which emphasizes the importance of POD prediction.

Previous studies have revealed age as a well-established predictor for POD with no certain mechanisms [16, 17]. In this present study, the patients who underwent POD also had a higher age than those without POD. However, the final multivariate analysis did not indicate age as a predicator as expected, which might be explained by the small age range or different operation types of the enrolled participants. Preoperative cognitive function decline is considered as a risk factor for postoperative cognitive problems and later life equality [18]. Our results from univariate analysis instead of multivariate analysis also showed a close association between preoperative MMSE score and POD. Previous studies have also indicated that longer operation and anesthesia time predict delirium after cardiac surgery due to increased cytokines release and operation complexity [19], which was not quite in accordance with our results.

Our results showed that patients with POD had higher CRP concentrations preoperatively and on postoperative day 2. However, the configured multivariate logistic regression model suggested plasma CRP concentrations as a predictor of POD preoperatively instead of postoperative day 2 . Therefore, preoperative plasma CRP concentrations may be an important risk biomarker for POD prediction in elderly patients with colon carcinoma after laparoscopic surgery. The pathophysiologic preexisted differences in the inflammatory activity during patients before the surgery might lead to different incidences of POD. This might support our findings from a pathophysiologic standpoint and offer new 
important targets for investigation. However, the association between preoperative CRP concentrations and POD still remains controversial until now with different conclusions in different patient samples. Previous literature examining the relationship between POD and CRP has suggested the potential predicative role of preoperative and postoperative CRP concentrations for POD in older patients undergoing major elective surgery [20]. Other reports conducted in small samples undergoing hip and vascular surgery showed positive associations between postoperative CRP concentrations and POD [21, 22], which is not so aligned with our results. In contrast to our findings, another two studies conducted in small cohorts observed no significant association between POD and preoperative CRP concentrations [12, 23]. No close correlations were observed in critically ill medical patients [24]. Different small sample sizes of cohort-based studies, different age ranges, different surgery types, and some other confounding factors may be potential explanations for the disparate conclusions between other previous reports and our study. Those individuals with a heightened inflammatory response are at greater risk of POD occurrence as proposed by current POD pathophysiology models [25]. With no welldefined etiology of multifactorial POD, the hypothesis of inflammatory processes leading to neuroinflammation has gained wide attraction in recent years [26].

This study has some limitations. First, this study is conducted in a single-center and has a relatively small sample size in comparison with other multicenter researches. Second, the group, age range, disease diagnosis, and operation types were all relatively specific. Furthermore, the inclusion criteria of this study were not so strict and some comorbidities (such as arthritis, infections, and inflammatory diseases) might affect the results. Last, why the involved mechanisms preoperative CRP concentrations can serve as a predicator for POD still remains unclear.

In conclusion, this present study highlighted the predictive role of preoperative CRP concentrations for POD in elderly patients undergoing laparoscopic surgery for colon carcinoma. Our evidence suggested its potential role for risk stratification before the surgery from a clinical point. More intensive assessments and preventive interventions could be recommended in those patients with high risk.

\section{Conflicts of Interest}

All the authors declare that they have no conflicts of interest.

\section{Authors' Contributions}

Dong Xiang and Hailin Xing participated in the conception and design, data collection, and statistical analysis and wrote the manuscript. Guozhu Xie and Huiyu Tai participated in the conception and design and data collection.

\section{References}

[1] M. Klugkist, B. Sedemund-Adib, C. Schmidtke, P. Schmucker, H. H. Sievers, and M. Hüppe, "Confusion Assessment Method for the Intensive Care Unit (CAM-ICU). Diagnosis of postoperative delirium in cardiac surgery," Der Anaesthesist, vol. 57, no. 5, pp. 464-474, 2008.

[2] E. W. Ely, A. Shintani, B. Truman et al., "Delirium as a predictor of mortality in mechanically ventilated patients in the intensive care unit," The Journal of the American Medical Association, vol. 291, no. 14, pp. 1753-1762, 2004.

[3] S. Ouimet, B. P. Kavanagh, S. B. Gottfried, and Y. Skrobik, "Incidence, risk factors and consequences of ICU delirium," Intensive Care Medicine, vol. 33, no. 1, pp. 66-73, 2007.

[4] S. T. Dillon, S. M. Vasunilashorn, L. Ngo et al., "Higher CReactive Protein Levels Predict Postoperative Delirium in Older Patients Undergoing Major Elective Surgery: A Longitudinal Nested Case-Control Study," Biological Psychiatry, vol. 81, no. 2, pp. 145-153, 2017.

[5] J. L. Rudolph and E. R. Marcantonio, "Postoperative Delirium: Acute change with long-term implications," Anesthesia \& Analgesia, vol. 112, no. 5, pp. 1202-1211, 2011.

[6] K. Franco, D. Litaker, J. Locala, and D. Bronson, "The cost of delirium in the surgical patient," Psychosomatics, vol. 42, no. 1 , pp. 68-73, 2001.

[7] J. C. Jackson, S. M. Gordon, R. P. Hart, R. O. Hopkins, and E. W. Ely, "The association between delirium and cognitive decline: A review of the empirical literature," Neuropsychology Review, vol. 14, no. 2, pp. 87-98, 2004.

[8] L. McNicoll, M. A. Pisani, Y. Zhang, E. W. Ely, M. D. Siegel, and S. K. Inouye, "Delirium in the intensive care unit: Occurrence and clinical course in older patients," Journal of the American Geriatrics Society, vol. 51, no. 5, pp. 591-598, 2003.

[9] S. E. de Rooij, B. C. van Munster, J. C. Korevaar, and M. Levi, "Cytokines and acute phase response in delirium," Journal of Psychosomatic Research, vol. 62, no. 5, pp. 521-525, 2007.

[10] J. Cerejeira, P. Batista, V. Nogueira, A. Vaz-Serra, and E. B. Mukaetova-Ladinska, "The stress response to surgery and postoperative delirium: Evidence of hypothalamic-pituitaryadrenal axis hyperresponsiveness and decreased suppression of the GH/IGF-1 axis," Journal of Geriatric Psychiatry and Neurology, vol. 26, no. 3, pp. 185-194, 2013.

[11] R. A. Pol, B. L. Van Leeuwen, G. J. Izaks et al., "C-reactive protein predicts postoperative delirium following vascular surgery," Annals of Vascular Surgery, vol. 28, no. 8, pp. 1923-1930, 2014.

[12] H. J. Lee, D. S. Hwang, S. K. Wang, I. S. Chee, S. Baeg, and J. L. Kim, "Early assessment of delirium in elderly patients after hip surgery," Psychiatry Investigation, vol. 8, no. 4, pp. 340-347, 2011.

[13] M. E. Charlson, P. Pompei, K. L. Ales, and C. R. MacKenzie, "A new method of classifying prognostic comorbidity in longitudinal studies: development and validation," Journal of Chronic Diseases, vol. 40, no. 5, pp. 373-383, 1987.

[14] E. W. Ely, R. Margolin, J. Francis et al., "Evaluation of delirium in critically ill patients: validation of the Confusion Assessment Method for the Intensive Care Unit (CAM-ICU)," Critical Care Medicine, vol. 29, no. 7, pp. 1370-1379, 2001.

[15] M. John, E. W. Ely, D. Halfkann et al., "Acetylcholinesterase and butyrylcholinesterase in cardiosurgical patients with postoperative delirium," Journal of Intensive Care, vol. 5, no. 29, 2017.

[16] M. C. Tan, A. Felde, M. Kuskowski et al., "Incidence and predictors of post-cardiotomy delirium," The American Journal of Geriatric Psychiatry, vol. 16, no. 7, pp. 575-583, 2008.

[17] A. Afonso, C. Scurlock, D. Reich et al., "Predictive model for postoperative delirium in cardiac surgical patients," Seminars in Cardiothoracic and Vascular Anesthesia, vol. 14, no. 3, pp. 212217,2010 
[18] J. L. Rudolph, R. N. Jones, S. E. Levkoff et al., "Derivation and validation of a preoperative prediction rule for delirium after cardiac surgery," Circulation, vol. 119, no. 2, pp. 229-236, 2009.

[19] B. Yildizeli, M. Oğuzhan Özyurtkan, H. F. Batirel, K. Kuşcu, N. Bekiroğlu, and M. Yüksel, "Factors associated with postoperative delirium after thoracic surgery," The Annals of Thoracic Surgery, vol. 79, no. 3, pp. 1004-1009, 2005.

[20] S. M. Vasunilashorn, S. T. Dillon, S. K. Inouye et al., "High C-reactive protein predicts delirium incidence, duration, and feature severity after major noncardiac surgery," Journal of the American Geriatrics Society, vol. 65, no. 8, pp. e109-e116, 2017.

[21] S. McGrane, T. D. Girard, J. L. Thompson et al., "Procalcitonin and C-reactive protein levels at admission as predictors of duration of acute brain dysfunction in critically ill patients," Critical Care, vol. 15, no. 2, article no. R78, 2011.

[22] C. W. Ritchie, T. H. Newman, B. Leurent, and E. L. Sampson, "The association between C-reactive protein and delirium in 710 acute elderly hospital admissions," International Psychogeriatrics, vol. 26, no. 5, pp. 717-724, 2014.

[23] A. W. Lemstra, K. J. Kalisvaart, R. Vreeswijk, W. A. van Gool, and P. Eikelenboom, "Pre-operative inflammatory markers and the risk of postoperative delirium in elderly patients," International Journal of Geriatric Psychiatry, vol. 23, no. 9, pp. 943-948, 2008.

[24] M. van den Boogaard, M. Kox, K. L. Quinn et al., "Biomarkers associated with delirium in critically ill patients and their relation with long-term subjective cognitive dysfunction; indications for different pathways governing delirium in inflamed and noninflamed patients," Critical Care, vol. 15, no. 6, article no. R297, 2011.

[25] E. R. Marcantonio, "Postoperative delirium," The Journal of the American Medical Association, vol. 308, no. 1, pp. 73-81, 2012.

[26] J. Cerejeira, H. Firmino, A. Vaz-Serra, and E. B. MukaetovaLadinska, "The neuroinflammatory hypothesis of delirium," Acta Neuropathologica, vol. 119, no. 6, pp. 737-754, 2010. 


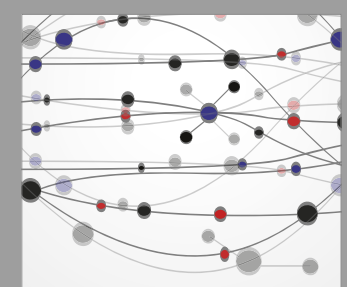

The Scientific World Journal
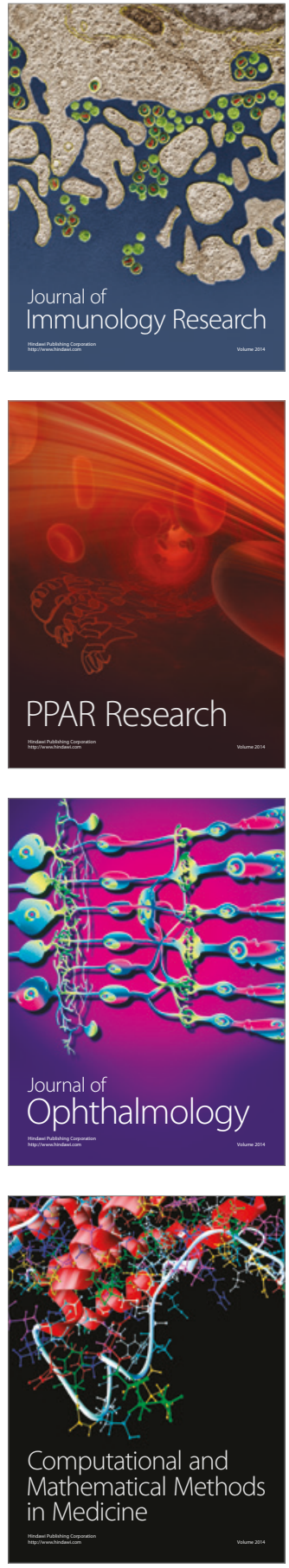

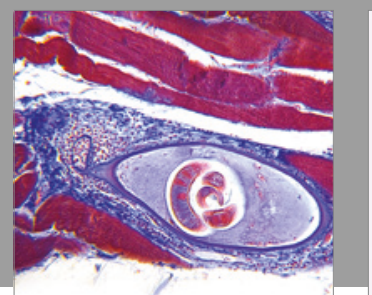

Gastroenterology Research and Practice
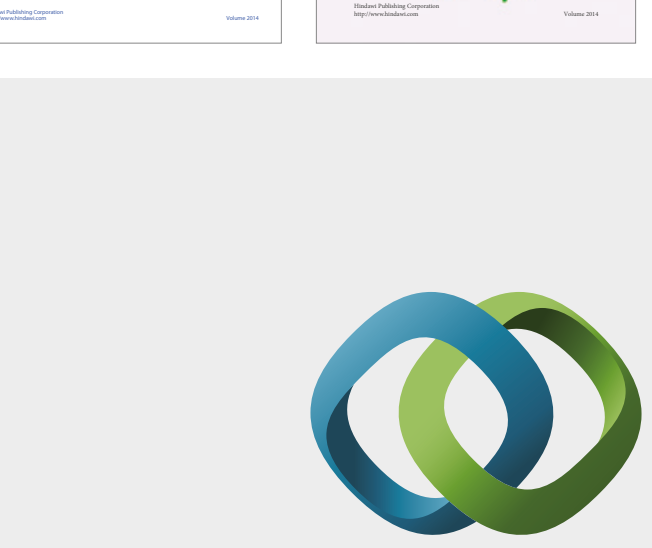

\section{Hindawi}

Submit your manuscripts at

https://www.hindawi.com
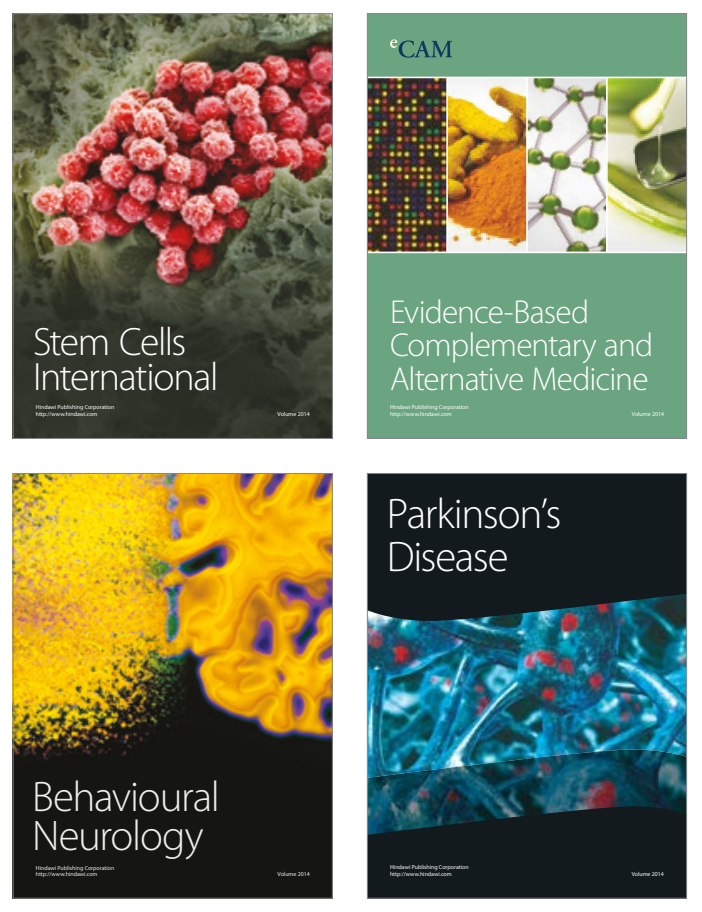
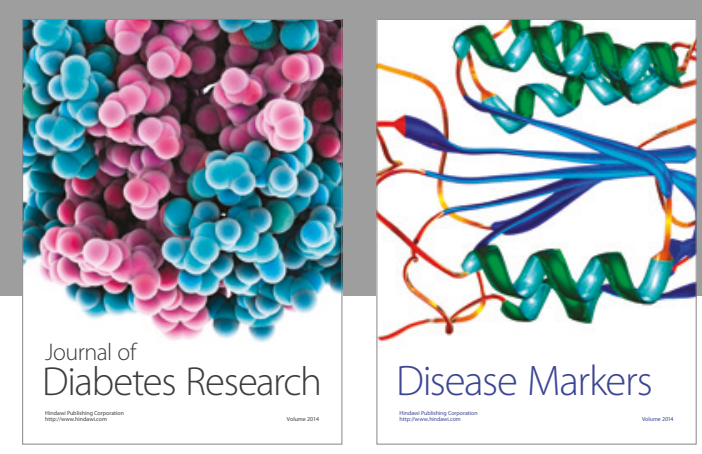

Disease Markers
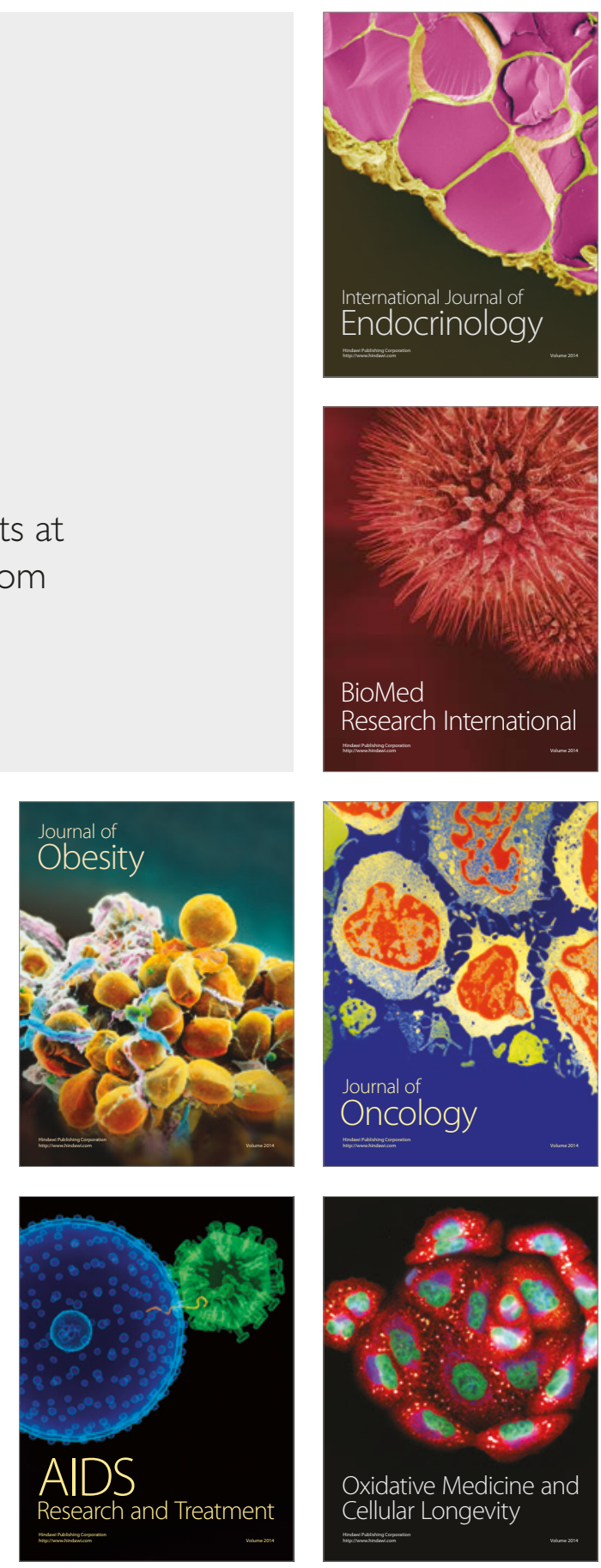